\section{Ça « chat » entre la microflore intestinale et l'hôte}

Alain L. Servin
Inserm, Université Paris-Sud, U756,

Faculté de Pharmacie,

rue Jean-Baptiste Clément,

92296 Châtenay-Malabry, France.

alain.servin@u-psud.fr

de transformation des nutriments non digestibles.

J.y. Gordon et al. ont récemment publié deux articles remarquables qui ont fait avancer de façon significative les connaissances sur la relation hôte/ microflore intestinale. Ces auteurs ont développé une élégante approche expérimentale en construisant un modèle de poisson-zèbre (Danio rerio) sans microflore [9]. Comme chez les rongeurs sans microflore, l'implantation, chez le poisson-zèbre sans microflore, d'une microflore non segmentée de poissonzèbre élevé dans des conditions conventionnelles accélère le renouvellement cellulaire de l'épithélium intestinal et favorise la différenciation entérocytaire. Une analyse par micro-arrays conduite chez le poisson-zèbre sans microflore et le poisson-zèbre colonisé par une flore de poisson-zèbre révèle que 212 gènes du tractus gastro-intestinal sont régulés par la microflore du poisson-zèbre. De façon intéressante, les auteurs ont montré que 59 des réponses dues à la microflore intestinale poisson-zèbre sont aussi observées lorsque la souris sans microflore est reconstituée avec une microflore de souris. Les gènes régulés codent pour des intermédiaires du métabolisme des nutriments et de l'immunité innée et pour le renouvellement cellulaire épithélial. Partant de ce constat, le groupe de J.Y. Gordon a récemment entrepris l'implantation croisée des microflores poisson-zèbre $(Z)$ ou souris $(S)$ chez le poisson-zèbre et la souris sans microflore afin d'obtenir des modèles Z-poissonzèbre et $\mathrm{S}$-poisson-zèbre, et des modèles 
Z-souris et S-souris [10]. L'analyse des animaux conventionnalisés $S$-poissonzèbre et $Z$-souris montre que les hôtes opèrent une sélection des espèces microbiennes à partir des microflores intestinales $S$ et $Z$ initiales. Par ailleurs, une analyse génomique GeneChip conduite sur la partie distale de l'intestin grêle des modèles $\mathrm{Z}$-souris et $\mathrm{S}$-souris montre que bien que les microflores poisson-zèbre et souris soient différentes, celles-ci induisent chez la souris une réponse remarquablement similaire: 500 réponses dues à la microflore souris et 525 réponses dues à la microflore poisson-zèbre. La moitié approximativement de ces gènes répond quelle que soit la microflore et $96,4 \%$ de ceux-ci sont régulés de la même façon. Parmi ces gènes, certains codent pour des protéines impliquées dans des fonctions métaboliques: biosynthèse et métabo- lisme des acides gras, métabolisme des acides aminés essentiels, métabolisme du butyrate, et biosynthèse des acides biliaires. L'ensemble de ces travaux montre que : (1) l'intestin fournit un habitat permettant la sélection d'espèces bactériennes pour la constitution de la microflore intestinale résidente; (2) que cet habitat constitué de niches écologiques, permet le développement différentiel par mutualisme et symbiose des espèces bactériennes résidentes; et (3) que la microflore résidente influe sur le développement et la fonctionnalité du tractus gastro-intestinal de l'hôte. $\diamond$

Cross-talk between

the gut microflora and the host

\section{RÉFÉRENCES}

1. Hooper LV, Gordon JI. Commensal host-bacterial relationships in the gut. Science 2001 ; 292 : 1115-8.
2. Ley RE, Peterson DA, Gordon JI. Ecological and evolutionary forces shaping microbial diversity in the human intestine. Cell $2006 ; 124: 837-48$.

3. Gill SR, Pop M, Deboy RT, et al. Metagenomic analysis of the human distal gut microbiome. Science 2006; 312: 1355-9.

4. Backhed F, Ley RE, Sonnenburg JL, et al. Host-bacterial mutualism in the human intestine. Science 2005 ; 307: 1915-20.

5. Samuel BS, Gordon JI. A humanized gnotobiotic mouse model of host-archaeal-bacterial mutualism. Proc Natl Acad Sci USA 2006 ; 103 : 10011-6.

6. Hooper LV, Wong MH, Thelin A, et al. Molecular analysis of commensal host-microbial relationships in the intestine. Science 2001 ; 291 : 881-4.

7. Xu J, Bjursell MK, Himrod J, et al. A genomic view of the human-Bacteroides thetaiotaomicron symbiosis. Science 2003 ; 299 : 2074-6.

8. Hooper LV, Midtvedt T, Gordon JI. How host-microbial interactions shape the nutrient environment of the mammalian intestine. Annu Rev Nutr 2002 ; 22 : 283-307.

9. Rawls JF, Samuel BS, Gordon JI. Gnotobiotic zebrafish reveal evolutionarily conserved responses to the gut microbiota. Proc Natl Acad Sci USA 2004 ; $101:$ 4596-601.

10. Rawls JF, Mahowald MA, Ley RE, Gordon JI. Reciprocal gut microbiota transplants from zebrafish and mice to germ-free recipients reveal host habitat selection. Cell $2006 ; 127: 423-33$.

\section{NOUVELLE}

\section{L'adhérence}

\section{guide la polarité cellulaire}

Manuel Théry, Michel Bornens

> La morphogenèse et le renouvellement des édifices biologiques impliquent la coordination d'un grand nombre de cellules qui adoptent des comportements collectifs. Cette coordination nécessite la cohérence mécanique et fonctionnelle de l'ensemble des cellules qui composent le tissu. Afin d'assurer cette cohérence, chaque cellule doit s'accorder précisément avec son environnement en adaptant son architecture et son organisation interne. L'identification des signaux biochimiques que les cellules échangent et des paramètres mécaniques auxquels elles sont sensibles pour définir leur organisation interne et leur polarité est donc une étape clé vers la compréhension des lois de construction des architectures multicellulaires. Bien que l'importance de l'adhérence cellu- laire $[1,2]$ et des forces exercées sur la cellule [3] au sein des tissus ait été mise en évidence, la façon dont ces paramètres influencent l'organisation interne des cellules reste à découvrir. Ce type d'étude est actuellement limité expérimentalement. II est en effet très difficile de manipuler ces paramètres dans les tissus.

\section{Contrôle}

\section{du microenvironnement cellulaire}

Les techniques de microfabrication par photolithographie et les traitements de surface permettent aujourd'hui d'imprimer des protéines d'adhérence cellulaire selon des motifs qui ont la taille des cellules et d'entourer ces motifs de polymères anti-adhésifs $[4,5]$. En contrôlant la géométrie de leur patron adhésif, on peut contrôler individuellement la forme des cellules en culture [5]. Ainsi, il a pu être montré que l'allongement dans une direction privilégiée de la forme de la cellule (anisotropie) était un facteur géométrique capable d'influencer l'orientation de la polarité cellulaire [6]. II faut cependant noter que la forme est supportée par des structures cellulaires: c'est par l'établissement de points d'ancrage avec l'environnement extracellulaire et par l'assemblage d'actine à partir de ces points que la cellule acquiert sa forme. In vivo, la localisation de ces points d'ancrage dépend de la disposition des cellules voisines et de la struc- 